\title{
A COHERENT SEMICLASSICAL TRANSPORT MODEL FOR PURE-STATE QUANTUM SCATTERING*
}

\author{
SHI JIN ${ }^{\dagger}$ AND KYLE A. NOVAK
}

Dedicated to the sixtieth birthday of Professor Andrew Majda

\begin{abstract}
We present a time-dependent semiclassical transport model for coherent pure-state scattering with quantum barriers. The model is based on a complex-valued Liouville equation, with interface conditions at quantum barriers computed from the steady-state Schrödinger equation. By retaining the phase information at the barrier, this coherent model adequately describes quantum scattering and interference at quantum barriers, with a computational cost comparable to that of classical mechanics. We construct both Eulerian and Lagrangian numerical methods for this model, and validate it using several numerical examples, including multiple quantum barriers.
\end{abstract}

Key words. multiscale method, semiclassical limit, Liouville, coherent, quantum barrier.

AMS subject classifications. 65M06, 65Z05, 81Q20, 81S30, 81T80.

\section{Introduction}

The motion of electrons in a plasma or a semiconductor can be modeled with classical mechanics when the change in the applied potential is moderate. But in a region where the potential changes rapidly over a length on the order of a de Broglie wavelength, quantum mechanics is required to accurately capture wave phenomena such as tunneling, resonance, and partial transmission and reflections. Because quantumscale parameters often control the accuracy and consistency of the solution, one often must resolve the dynamics entirely at the quantum scale. But for large-scale problems, such an approach is numerically infeasible. If the quantum region is sufficiently localized, a viable approach is to solve the problem using a multiscale method that combines the large-scale classical model with the small-scale quantum model.

In $[7,8]$ the authors presented a multiscale approach which accurately models the interaction of a quantum wave packet with a thin barrier in the semiclassical regime as the scaled Planck constant $\varepsilon$ vanishes. This thin-barrier model accurately describes the weak limit of the moments of solutions to the pure-state Schrödinger and mixedstate von Neumann equation for an isolated thin quantum barrier (a barrier of width on the order of a de Broglie wavelength). This model assumes that the dwell time of the particle in the barrier is sufficiently short so that the behavior of the wave packet may be adequately described by the steady-state Schrödinger equation. Such an assumption is realistic for $O(\varepsilon)$ thin barriers in the semiclassical limit as $\varepsilon \rightarrow 0$, but it is inadequate when either $\varepsilon$ is finite or the width of the barrier is significant in comparison to the width of the wave packet.

Another shortcoming of the thin-barrier model is that it can only generate a decoherent solution. Quantum mechanics is in essence wave mechanics and the Schrö-

* Received: Decemober 27, 2008; accepted (in revised version): January 30, 2009.

This research was supported by NSF grant No. DMS-0608720 and NSF FRG grant DMS-0757285. Shi Jin was also supported by a Van Vleck Distinguished Research Prize for University of Wisconsin.

†Department of Mathematics, University of Wisconsin, 480 Lincoln Drive, Madison, Wisconsin 53706-1338, USA (jin@math.wisc.edu).

$\ddagger$ Department of Mathematics and Statistics, Air Force Institute of Technology, 2950 Hobson Way, Wright-Patterson AFB, Ohio, 45433-7765, USA (kyle.novak@afit.edu).

The views expressed in this article are those of the author and do not reflect the official policy or position of the United States Air Force, Department of Defense, or the U.S. Government. 
dinger equation models the evolution of waves. Two or more waves can constructively and deconstructively interfere over large distances and after long periods of time. Such behavior, called coherence, is destroyed by random media leading to decoherence in the classical solution. A model that only captures the decoherent behavior is adequate for many physical problems, but several devices such as superconductors and theoretical quantum computers rely on quantum coherence. Furthermore, a decoherent solution cannot adequately model a periodic crystalline lattice for which interference plays a key role.

In this article, we extend the thin-barrier model by proposing a coherent model that includes complex phase information. It is based on the complex Liouville equation with suitable interface conditions at quantum barrier to account for quantum scattering information. The interface conditions use complex-valued quantum scattering matrices computed by solving the steady-state Schrödinger equation, thus retaining the phase information needed for interference of waves that pass through resonant quantum barriers.

section 2 reviews the correspondence between classical and quantum mechanics. We then describe the semiclassical model in section 3 by reviewing the thin-barrier model, examining its limitations, and presenting the new coherent semiclassical model. In section 4 we discuss Eulerian and Lagrangian implementations of the model. Finally, section 5 presents several examples, including multiple resonant quantum barriers, to validate the model and verify the numerical method. Our results indicate that the model correctly captures the solution of the Schrödinger equation in the entire domain in the semiclassical limit, including interference at the barriers. Since the construction of quantum scattering information is at the pre-processing step, the overall computational cost using the complex-valued Liouville equation or Hamiltonian system is almost the same as that of using classical mechanics.

\section{Background}

The trajectory of a particle such as an electron can be modeled by the classical Hamiltonian system

$$
\frac{d x}{d t}=\frac{\partial H}{\partial p}, \quad \frac{d p}{d t}=-\frac{\partial H}{\partial x}
$$

where the Hamiltonian $H(x, p)=\frac{1}{2} m^{-1} p^{2}+V(x)$ gives the total energy and $x$ denotes the position, $p$ denotes the momentum, and $m$ is the effective mass. By the Liouville theorem, the probability distribution $f(x, p, t)$ of a particle is merely advected along the characteristics. Hence from (2.1), the time evolution of a distribution of noninteracting particles is given by the Liouville equation

$$
\frac{d f}{d t}=\frac{\partial f}{\partial t}+\frac{p}{m} \frac{\partial f}{\partial x}-\frac{d V}{d x} \frac{\partial f}{\partial p}=0 .
$$

The dynamics of a particle can also be modeled using the Schrödinger equation

$$
i \hbar \frac{\partial}{\partial t} \psi=\hat{H} \psi=\left(-\frac{\hbar^{2}}{2 m} \frac{d^{2}}{d x^{2}}+V(x)\right) \psi,
$$

where $\hbar$ is Planck's constant. The Schrödinger equation describes the time evolution of the probability amplitude $\psi(x, t)$. When the potential is sufficiently smooth, the probability density $\rho(x, t)=|\psi(x, t)|^{2}$ in the semiclassical limit as $\varepsilon \rightarrow 0$ may adequately be determined by the classical Liouville equation. Consider a characteristic length 
and time scale $L \delta x$ and $L \delta t$ where $\delta x$ is the natural length scale such as a de Broglie wavelength $\delta x=\hbar / p$ for some momentum $p$. By rescaling $x \mapsto x / L \delta x$ and $t \mapsto t / L \delta t$ in the Schrödinger equation we have

$$
i \varepsilon \frac{\partial}{\partial t} \psi=\hat{H} \psi=\left(-\frac{\varepsilon^{2}}{2 m} \frac{d^{2}}{d x^{2}}+V(x)\right) \psi
$$

where the dimensionless scaled Planck constant $\varepsilon=\left[m L(\delta x)^{2} / \delta t\right]^{-1} \hbar$ and the effective mass $m$ has been nondimensionalized. The Wigner transform is defined as

$$
W(x, p, t)=\frac{1}{2 \pi} \int_{-\infty}^{\infty} \psi\left(x+\frac{1}{2} \varepsilon y, t\right) \bar{\psi}\left(x-\frac{1}{2} \varepsilon y, t\right) e^{-i p y} d y .
$$

By applying the transform to the Schrödinger equation one has the Wigner equation $[28]$

$$
\frac{\partial}{\partial t} W+\frac{p}{m} \frac{\partial W}{\partial x}-\Theta^{\varepsilon} W=0
$$

where the nonlocal term is

$$
\Theta^{\varepsilon} W(x, p, t)=\frac{1}{2 \pi} \int_{-\infty}^{\infty} \frac{i}{\varepsilon}\left[V\left(x+\frac{1}{2} \varepsilon y\right)-V\left(x-\frac{1}{2} \varepsilon y\right)\right] \check{W}(x, y, t) e^{-i p y} d y,
$$

with

$$
\check{W}(x, y, t)=\int_{-\infty}^{\infty} W(x, p, t) e^{i p y} d p
$$

being the Fourier transform of $W(x, p, t)$. When the potential $V(x)$ is sufficiently smooth, one recovers the classical Liouville Equation (2.2) in the semiclassical limit as $\varepsilon \rightarrow 0[5,18]$. It is important to note that the Wigner function $W(x, p, t)$ is realvalued and and does not correspond to a unique wavefunction $\psi(x, t)$. The Wigner transform of $\psi(x, t) e^{i \theta}$ for any $\theta$ produces the same function $W(x, p, t)$. Because of this, the Liouville equation cannot adequately model important wave phenomena. In this article, we introduce a complex Liouville equation, which retains the phase information. Formally, we define a new complex function $\Phi(x, p, t)=\sqrt{W(x, p, t)} e^{i \theta}$ that both retains the linear superpositioning characteristic of $\psi(x, t)$ and satisfies the Liouville equation.

Furthemore, the classical limit is not valid at discontinuities in the potential $[3,23,25]$. Instead, the potential behaves as a quantum scatterer. Instead, one may consider a multiscale domain decomposition approach for a solution [4]. The next section presents a semiclassical model for a thin quantum barriers.

\section{Semiclassical models}

This section discusses the semiclassical models. We begin by presenting an overview of the thin-barrier model which was developed in $[7,8]$ for quantum barriers or wells that are sufficiently thin in comparison to the support of the wavepacket. The thin-barrier model fails to capture important phenomena such as interference. Therefore, we extend the thin-barrier model to the coherent semiclassical model. 
3.1. A thin-barrier model. Consider the Hamiltonian system (2.1). The characteristic of the function $H(x, p)$ is the integral curve $\varphi(t)=(x(t), p(t))$. Note that $\varphi(t)$ may not be defined for all time $t \in \mathbb{R}$. When $H(\varphi(t))$ is differentiable,

$$
\frac{d}{d t} H(\varphi(t))=\frac{d x}{d t} \frac{\partial H}{\partial x}+\frac{d p}{d t} \frac{\partial H}{\partial t}=0
$$

from which it follows that the Hamiltonian is constant along any characteristic $\varphi(t)$, i.e.,

$$
H(\varphi(t))=\frac{1}{2} m^{-1} p^{2}+V(x)=E .
$$

Equation (3.1) is the strong form of the conservation of energy and equation (3.2) is the weak form. If the potential $V(x)$ is discontinuous or not defined in the semiclassical limit in some region $\mathcal{Q}$, the Hamiltonian system fails to have a global solution. In such cases, it is appropriate to use a Hamiltonian-preserving scheme.

The key idea behind the Hamiltonian-preserving schemes $[9,10,11]$ is (a) to solve the Liouville equation locally in regions where the gradient of $V(x)$ exists and (b) to use the weak form of the conservation of energy to connect the local solutions together across a barrier using an interface condition that captures the correct transmissions and reflections. By interpreting a wavefunction as a statistical ensemble of a large number of particles [22], we may model the quantum solution using semiclassical characteristics.

Let $\mathcal{L}$ be the locally defined set of characteristics of the function $H(x, p)$. By requiring the Hamiltonian to be constant along trajectories, we create an equivalence class of characteristics $[\varphi]=\left\{\varphi^{*} \in \mathcal{L} \mid H\left(\varphi^{*}\right)=H(\varphi)\right\}$. In one dimension, an incident characteristic may be connected to a reflected characteristic or a transmitted characteristic. Let $\left(x_{\mathrm{in}}, p_{\mathrm{in}}\right)$ be the limit of an incoming trajectory on the barrier and $\left(x_{\text {out }}, p_{\text {out }}\right)$ be the limit of an outgoing trajectory at the barrier. From the conservation of energy (3.2), the momenta for a reflected particle is

$$
p_{\text {out }}=-p_{\text {in }}
$$

while the momenta for a transmitted particle is

$$
p_{\text {out }}=p_{\text {in }} \sqrt{1+2 m\left[V\left(x_{\text {in }}\right)-V\left(x_{\text {out }}\right)\right] / p_{\text {in }}^{2}} .
$$

If $p_{\text {in }}^{2}<2 m\left[V\left(x_{\mathrm{in}}\right)-V\left(x_{\mathrm{out}}\right)\right]$, the momentum of the transmitted particle is imaginary, and the particle is always reflected. Furthermore, particles may be split into reflected and transmitted particles as long as the total probability density $f(x, p, t)$ is conserved:

$$
f_{1, \text { out }}+f_{2, \text { out }}=f_{1, \text { in }}+f_{2, \text { in }} .
$$

where $f_{1, \text { in }}$ is the incident probability density from the left, $f_{2, \text { in }}$ is the incident probability density from the right, $f_{1, \text { out }}$ is the outgoing probability density to the left, and $f_{2 \text {,out }}$ is the outgoing probability density to the right. To resolve such nonuniqueness, we impose an additional interface condition. For thin quantum barriers, the interface condition can be derived from the time-independent Schrödinger equation across the interface assuming the following conditions:

1. The effective width of a barrier is on the order of a de Broglie wavelength and the barrier dwell time is $O(\varepsilon)$. 
2. Each barrier is considered independently of every other barrier. That is, the coherence time is sufficiently short and therefore interference away from the barrier may be neglected.

These assumptions impose several limitations on the model which we will address in section 3.2. Removal of these assumptions is the focus of this article.

The interface condition is given by the scattering coefficients to the timeindependent Schrödinger equation. The scattering coefficients may be computed by considering the barrier as an open quantum system [2] outside of which the potential is constant. Typically, one may use a quantum transmitting boundary method [15], a spectral projection method [19], or a transfer matrix method $[1,13,6]$. In these approaches, one separates the domain into three regions, $\mathcal{C}_{1}, \mathcal{Q}$, and $\mathcal{C}_{2}$. Let the potential $V(x)$ be constant $V_{1}$ and $V_{2}$ in regions $\mathcal{C}_{1}$ and $\mathcal{C}_{2}$, respectively. For an energy $E$ the time-independent Schrödinger equation

$$
-\frac{\varepsilon^{2}}{2 m} \psi^{\prime \prime}(x)+V(x) \psi(x)=E
$$

has the solution

$$
\psi(x)= \begin{cases}a_{1} e^{i p_{1} x / \varepsilon}+b_{1} e^{-i p_{1} x / \varepsilon}, & x \in \mathcal{C}_{1}, \\ \psi_{\mathcal{Q}}, & x \in \mathcal{Q}, \\ a_{2} e^{i p_{2} x / \varepsilon}+b_{2} e^{-i p_{2} x / \varepsilon}, & x \in \mathcal{C}_{2}\end{cases}
$$

where $p_{1,2}=\sqrt{2 m\left(E-V_{1,2}\right)}$ and the coefficients $a_{1}, a_{2}, b_{1}$, and $b_{2}$ are uniquely determined by the boundary conditions at $x_{1}$ and $x_{2}$.

By assuming the matching conditions that $\psi(x)$ and its derivative are continuous, $\psi_{\mathcal{Q}}$ is uniquely determined by the values $a_{1}$ and $b_{1}$ using the boundary in regions $C_{1}$. Thus, the coefficients are equated by a scattering matrix

$$
\left(\begin{array}{l}
b_{1} \\
a_{2}
\end{array}\right)=\left(\begin{array}{ll}
r_{1} & t_{2} \\
t_{1} & r_{2}
\end{array}\right)\left(\begin{array}{l}
a_{1} \\
b_{2}
\end{array}\right)
$$

The time evolution of the position density $\rho(x, t)=|\psi(x, t)|^{2}$ in the Schrödinger equation, gives the continuity equation

$$
\frac{\partial \rho}{\partial t}+\frac{d J}{d x}=0
$$

where the current-density

$$
J(x)=\varepsilon m^{-1} \operatorname{Im}\left(\bar{\psi} \frac{\partial}{\partial x} \psi\right)= \begin{cases}p_{1}\left(\left|a_{1}\right|^{2}-\left|b_{1}\right|^{2}\right) / m, & x \in \mathcal{C}_{1} \\ p_{2}\left(\left|a_{2}\right|^{2}-\left|b_{2}\right|^{2}\right) / m, & x \in \mathcal{C}_{2}\end{cases}
$$

The positive-valued terms of $J(x)$ give the flux of right-traveling waves and the negative-valued terms give the flux of left-traveling waves. In particular, for a wave incident on the barrier from the left $\left(b_{2} \equiv 0\right)$, we have $a_{2}=t_{1} a_{1}$ and $b_{1}=r_{1} a_{1}$. It follows that the reflection coefficient $R$, the ratio of the reflected to incident current densities, and the transmission coefficient $T$, the ratio of the transmitted to incident current densities, are

$$
R=\left|r_{1}\right|^{2} \quad \text { and } \quad T=\left(p_{2} / p_{1}\right)\left|t_{1}\right|^{2}
$$




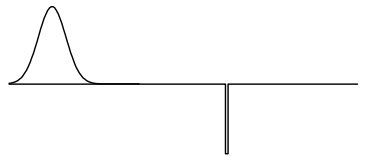

(a)

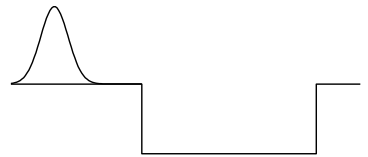

(b)

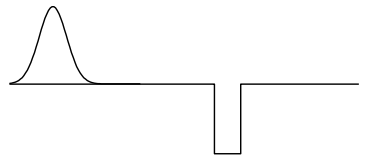

(c)

FIG. 3.1. Illustration of a wavepacket juxtapositioned with three barriers: a thin barrier, a wide barrier and a mesoscopic barrier.

A wave of the same energy incident from the right has the same scattering coefficients. Hence, the real-valued interface condition used to incorporate reflection and transmission is

$$
\begin{aligned}
& f_{1, \text { out }}=R f_{1, \text { in }}+T f_{2, \text { in }} \\
& f_{2, \text { out }}=T f_{1, \text { in }}+R f_{2, \text { in }}
\end{aligned}
$$

To implement the model, solve the Liouville Equation (2.2) using the interface conditions given by (3.8) by matching (3.3a) and (3.3b). For a complete description of the one and two-dimensional thin-barrier models, see $[7,8]$.

3.2. Limitations of the thin-barrier model. As discussed in the previous section, the thin-barrier model divides a domain into two classical regions separated by a thin quantum barrier. If the barrier is sufficiently thin and the barrier dwell time is sufficiently short so that it vanishes in the semiclassical limit $\varepsilon \rightarrow 0$, then it is permissible to consider instantaneous transmission across the barrier. In such a case, the stationary Schrödinger equation adequately describes barrier transmission and reflection. This approximation is valid in the semiclassical limit if the effective width of a barrier is $O(\varepsilon)$ and interactions with each barrier are considered independent. A thin rectangular well (figure 3.1a) can be modeled using a single thin-barrier interface separating two classical regions. Similarly, a wide rectangular well (figure 3.1b) can be modeled using two independent interfaces separating three classical regions, because the wavepacket only interacts with one edge at a time. But the thin-barrier assumption fails for a rectangular well of a width on the order of the width of the wavepacket. The single-thin-barrier model is inappropriate because the transmission time across the well is sufficiently long and hence the steady-state Schrödinger equation is inadequate. Using two independent thin barriers will also give an incorrect solution because wave scattering at both edges of the well is coupled through interference. In this case, a more general model is needed.

A second shortcoming of the thin-barrier model is that it does not accurately measure barrier dwell time. To implement barrier dwell in the thin-barrier model, one can use the Wigner time delay, the delay to the group velocity of a wave packet. The reflection and transmission group delay times for unit mass are given by $\varepsilon p^{-1} d(\arg s) / d x$, where $s$ is either the complex-valued reflection or transmission coefficient [22]. Such an approach is useful only when the reflected and transmitted wave packets maintain well-defined peaks, which is not typical when the barrier is sufficiently wide. If the wavefunction envelope changes while the particle is trapped in the barrier, then the probability amplitudes cannot be approximated as being constant in time and the stationary Schrödinger equation cannot adequately describe barrier transmission and reflection. 
A third limitation is that, unlike the Liouville equation and pure-state Schrödinger equation, the thin-barrier model is entropy increasing and hence time-irreversible. The entropy of a system is given by $S[f](t)=-\iint_{-\infty}^{\infty} f \log f d x d p$ where $f(x, p, t)$ gives the probability density of a particle at $(x, p)$. As a consequence of the Liouville theorem the entropy is conserved $d S / d t=0$, as long as the potential $V(x)$ is differentiable. Since $S[f]$ is a convex-down function of $f, S\left[R f_{1}+T f_{2}\right] \geq R S\left[f_{1}\right]+T S\left[f_{2}\right]$ for $0 \leq R, T \leq 1$. So, by combining solutions along the characteristics, the entropy increases.

We can correct these shortcomings of the thin-barrier model by including phase information and, if necessary, dividing a barrier into several thin barriers. We discuss this approach in the next section.

3.3. A coherent semiclassical model. By linearity of Schrödinger's equation, two wave functions $\psi_{1}$ and $\psi_{2}$ with respective position densities $\rho_{1}$ and $\rho_{2}$, are superpositioned as a result of a transmission and reflection. The resultant position density is

$$
\rho_{1+2}=\left|\psi_{1}+\psi_{2}\right|^{2}=\rho_{1}+\rho_{2}+2 \sqrt{\rho_{1} \rho_{2}} \cos \theta,
$$

where $\theta=\arg \left(\psi_{1}-\psi_{2}\right)$ is a measure of the phase difference of two wave functions. To account for the nonlinear interference with respect to the probability densities, we similarly take the coherent semiclassical interface condition as

$$
\begin{aligned}
& f_{1, \text { out }}=R f_{1, \text { in }}+T f_{2, \text { in }}+2 \sqrt{R T f_{1, \text { in }} f_{2, \text { in }}} \cos \theta \\
& f_{2, \text { out }}=T f_{1, \text { in }}+R f_{2, \text { in }}-2 \sqrt{R T f_{1, \text { in }} f_{2, \text { in }}} \cos \theta
\end{aligned}
$$

where $\theta$ is the phase difference between the incident terms $f_{1, \text { in }}$ and $f_{2, \text { in }}$. The Liouville Equation (2.2) is solved away from the barrier with this new interface condition. Note that the interface condition (3.9) merely requires the change in phase before and after transmission and reflection. The phase shift $\theta$ along a characteristic defined by the Hamiltonian $E$ is given by $\int_{\varphi(E)} p / \varepsilon d s$. Alternatively, the phase shift can be derived as the argument of the complex scattering coefficients determined by solving the time-independent Schrödinger equation across the barrier and in the classical region. The coherent model does not incorporate tunneling, for which the evanescent wave corresponds to an imaginary momentum. Such cases can be modeled by using the thin-barrier model over the entire barrier for those energies.

Since (3.9) is difficult to implement numerically, we propose an alternative, equivalent coherent semiclassical model. We impose a linear interface condition by defining the complex semiclassical amplitude $\Phi(x, p, t)$ such that $f(x, p, t)=|\Phi(x, p, t)|^{2}$ by taking

$$
\Phi(x, p, t)=\sqrt{f(x, p, t)} e^{i \theta(x, p)}
$$

where $\theta(x, p)$ is the phase difference from the initial conditions. In this case, from (3.6) and (3.7) the correct matching conditions are

$$
\begin{aligned}
& \Phi_{1, \text { out }}=\hat{r}_{2} \Phi_{1, \text { in }}+\hat{t}_{2} \Phi_{2, \text { in }}=r_{1} \Phi_{1, \text { in }}+\sqrt{\frac{p_{1}}{p_{2}}} t_{2, \text { in }} \Phi_{2, \text { in }} \\
& \Phi_{2, \text { out }}=\hat{t}_{1} \Phi_{1, \text { in }}+\hat{r}_{1} \Phi_{2, \text { in }}=\sqrt{\frac{p_{2}}{p_{1}}} t_{1, \text { in }} \Phi_{1, \text { in }}+r_{2} \Phi_{2, \text { in }}
\end{aligned}
$$

where $\Phi_{1, \text { in }}$ and $\Phi_{2, \text { in }}$ are the left and right limits of the solution incident on the barrier with momenta $p_{1}$ and $p_{2}$, respectively. Furthermore, if $d \Phi / d t=0$, then the 
Liouville condition $d f / d t=0$ follows directly. Hence, we solve

$$
\Phi_{t}+p \Phi_{x}-V_{x} \Phi_{p}=0
$$

in the classical region using the interface condition (3.10) at the quantum barrier.

Each of these two approaches (either solving $f$ or solving $\Phi$ ) presents several numerical implementation difficulties. In the first approach, to implement (3.9) we need to determine $\theta$. In the second approach, $\rho(x, t)=\int f(x, p, t) d p$ is not a conserved quantity. Furthermore, numerical viscosity introduces decoherence. Because the phase shift changes as $d \theta / d p \sim 1 / \varepsilon$, even a small numerical viscosity, which leads to the mixing of the cells of different momenta, results in a substantial error in the phase shift. Maintaining the correct phase shift is critical for the coherent model. We address these concerns in the next section.

\section{Numerical implementation}

This section presents the numerical implementation of the Eulerian formulation of the coherent model using a finite volume method and the implementation of the Lagrangian formulation using a particle/ray-tracing method. Each numerical approach has advantages and disadvantages. For example, the Lagrangian approach is a robust method that can easily be extended to higher dimensions. One or two-barrier problems may often be solved analytically by tracking all possible characteristics. But several barriers typically require a Monte Carlo splitting along the characteristics. Even in one dimension, the Monte Carlo Lagrangian method provides a noisy solution with low order convergence. The Eulerian approach gives a consistent solution but it cannot in the authors' opinion be extended beyond one dimension without substantial changes. For more complex systems, one might choose between Lagrangian and Eulerian approaches to simplify integration with the rest of the system.

4.1. Eulerian approach. One can either solve (2.2) with interface conditions (3.9) to determine $f(x, p, t)$ or solve (3.11) with interface conditions (3.10) to determine the phase shift $\theta$. When the momentum is discontinuous across an interface, the flux from incident cells must be separated and recombined into the transmitted cells $[9,7]$. For nonconstant potentials, the flux from incident cells must be separated and recombined in the transmitted cells. This mixing between characteristics leads to numerical viscosity, and because $d \theta=d p / \varepsilon$, it ultimately degrades the coherence in the solution when $\varepsilon$ is small. We simplify the implementation for one-dimensional problems by solving over the $(x, E)$-domain instead of the $(x, p)$-domain. In either case, discontinuities in $V(x)$ result in discontinuous momenta $p$ along the solution characteristics, but the energy $E$ remains continuous along the solution characteristics. Because the Hamiltonian $E$ is constant along any characteristic, we simplify implementation of the Liouville equation by assigning

$$
F(x, E, t) \equiv f(x, p, t)
$$

with $E=\frac{1}{2} p^{2}+V(x)$. Then

$$
\frac{d F}{d t}=\frac{\partial F}{\partial t}+p \frac{\partial F}{\partial x}=0
$$

where $p(x)= \pm \sqrt{2(E-V(x))}$. In conservation form,

$$
\frac{\partial Q}{\partial t}+\frac{\partial}{\partial x}(p Q)=0 \quad \text { with } \quad Q(x)=F(x) / p(x) .
$$


From (3.10) using $\Phi=\sqrt{Q p} e^{i \theta}$ it follows that the interface condition is

$$
\begin{gathered}
Q_{1, \text { out }}=\left|r_{1} \sqrt{Q_{1, \text { in }}} e^{i \theta_{1}}+t_{2} \sqrt{Q_{2, \text { in }}} e^{i \theta_{2}}\right|^{2} \\
Q_{2, \text { out }}=\left|t_{1} \sqrt{Q_{1, \text { in }}} e^{i \theta_{1}}+r_{2} \sqrt{Q_{2, \text { in }}} e^{i \theta_{2}}\right|^{2},
\end{gathered}
$$

where $e^{i \theta_{1}}=\Phi_{1, \text { in }} /\left|\Phi_{1, \text { in }}\right|$ and $e^{i \theta_{2}}=\Phi_{2, \text { in }} /\left|\Phi_{2, \text { in }}\right|$ are computed by solving $\Phi$. Note,

$$
\rho(x, t)=\int_{\Omega(x, p)} f(x, p, t) d p=\int_{\Omega(x, E)} F(x, E, t) \frac{d E}{p}=\int_{\Omega(x, E)} Q d E .
$$

Define the complex-valued function

$$
S(x, E, t)=\Phi(x, E, t) / p(x)
$$

which satisfies the (complex-valued) Liouville equation:

$$
\frac{\partial}{\partial t} S+\frac{\partial}{\partial x}(p S)=0
$$

with interface conditions (3.10)

$$
\begin{aligned}
& S_{1, \text { out }}=r_{1} S_{1, \text { in }},+\sqrt{\frac{p_{1}}{p_{2}}} t_{2} S_{2, \text { in }} \\
& S_{2, \text { out }}=\sqrt{\frac{p_{2}}{p_{1}}} t_{1} S_{1, \text { in }}+r_{2} S_{2, \text { in }} .
\end{aligned}
$$

A vanishing momentum may lead to loss of precision and care must be taken to avoid a mesh point at which $p(x)= \pm \sqrt{2(E-V(x)} \approx 0$. We only divide by $p(x)$ to transform the initial conditions and to enforce the interface condition (4.4), in which the transmission probability is also small if $p(x)$ is small.

Consider a uniform mesh $\left\{x_{i}\right\}$ and grid spacing $\Delta x$. Define a cell $C_{i}=$ $\left[x_{i-1 / 2}, x_{i+1 / 2}\right)$ ensuring that a cell boundary does not coincide with $p\left(x_{i \pm 1 / 2}\right)=0$. We shall consider the quantum barrier to be located at a cell interface $x_{Z+1 / 2}$ for some integer(s) $Z$. Define the cell average over the cell $C_{i}$ as

$$
Q_{i}^{n}=\frac{1}{\Delta x} \int_{C_{i}} Q\left(x, E, t_{n}\right) d x
$$

The finite-volume discretization of the Liouville Equation (4.1) is

$$
Q_{i}^{n+1}=Q_{i}^{n}-\frac{\Delta t}{\Delta x}\left[(p Q)_{i+1 / 2}^{-}-(p Q)_{i-1 / 2}^{+}\right]
$$

with the limits of $F=p Q$ defined by

$$
(p Q)_{i+1 / 2}^{ \pm}=\lim _{x \rightarrow x_{i+1 / 2}^{ \pm}} p(x, E) Q^{n}(x, E) .
$$

Upwinding is used to approximate the fluxes $Q_{i+1 / 2}^{ \pm}$. The finite-volume approximation for $S_{i}^{n}$ is defined similarly. 
The left and right limits of $Q$ and $S$ in the cells immediately downwind of the quantum barrier are determined by the interface condition

$$
\left.\begin{array}{l}
Q_{\text {out }}^{+}=\left|r_{2}(E) \frac{S_{\text {in }}^{+}}{\left|S_{\text {in }}^{+}\right|} \sqrt{Q_{\text {in }}^{+}}+t_{1}(E) \frac{S_{\text {in }}^{-}}{\left|S_{\text {in }}^{-}\right|} \sqrt{Q_{\text {in }}^{-}}\right|^{2} \\
S_{\text {out }}^{+}=r_{2}(E) S_{\text {in }}^{+}+\sqrt{\frac{p_{2}}{p_{1}}} t_{1}(E) S_{\text {in }}^{-} \\
Q_{\text {out }}^{-}=\left|t_{2}(E) \frac{S_{\text {in }}^{+}}{\left|S_{\text {in }}^{+}\right|} \sqrt{Q_{\text {in }}^{+}}+r_{1}(E) \frac{S_{\text {in }}^{-}}{\left|S_{\text {in }}^{-}\right|} \sqrt{Q_{\text {in }}^{-}}\right|^{2} \\
S_{\text {out }}^{-}=\sqrt{\frac{p_{1}}{p_{2}}} t_{2}(E) S_{\text {in }}^{+}+r_{1}(E) S_{\text {in }}^{-}
\end{array}\right\} \text {for } p>0
$$

For a first-order method, we take the approximation $Q_{i \mp 1 / 2}^{ \pm}=Q_{i}$ and $S_{i \mp 1 / 2}^{ \pm}=$ $S_{i}$. For a second-order method we use a flux-limited piecewise-linear interpolant to approximate the right and left limits

$$
Q_{i \mp 1 / 2}^{ \pm}=Q_{i} \mp \frac{1}{2}(1-\lambda) \sigma\left(Q_{i-1}, Q_{i}, Q_{i+1}\right)
$$

where $\lambda=\Delta t / \Delta x$ and the slope $\sigma(\cdot)$ is calculated using a minmod limiter [16]. We use a similar approximation for $S_{i \mp 1 / 2}^{ \pm}$. See [7].

4.2. Lagrangian approach. Analogously to the thin-barrier model, we define the semiclassical probability amplitude as the linear superposition

$$
\Phi(x, p, t)=\sum_{k} s_{k}(H(x, p)) \Phi_{k}(x, p, t)
$$

where

$$
\Phi_{k}(x, p, t)=\int \Phi(\tilde{x}, \tilde{p}, 0) \varphi_{k}(x, p, t ; \tilde{x}, \tilde{p}) d \tilde{x} d \tilde{p}
$$

is the solution along the $k$-th characteristic for the Hamiltonian $H(\tilde{x}, \tilde{p})$ with

$$
\varphi_{k}(x, p, t ; \tilde{x}, \tilde{p})=\delta(x(t)-\tilde{x}) \delta(p(t)-\tilde{p}) .
$$

The scattering term $s_{k}(H(x, p))$ is defined as the product of complex-valued transmission and reflection coefficients along the $k$ th characteristic:

$$
\left(\begin{array}{l}
\Phi_{1, \text { out }} \\
\Phi_{2, \text { out }}
\end{array}\right)=\left(\begin{array}{cc}
\hat{r}_{1} & \hat{t}_{2} \\
\hat{t}_{1} & \hat{r}_{2}
\end{array}\right)\left(\begin{array}{l}
\Phi_{1, \text { in }} \\
\Phi_{2, \text { in }}
\end{array}\right) .
$$

The coherent probability density is given by

$$
f(x, p, t)=\left|\sum_{k} s_{k}(H(x, p)) \Phi_{k}(x, p, t)\right|^{2}
$$

and the decoherent probability denity is given by

$$
f(x, p, t)=\sum_{k}\left|s_{k}(H(x, p)) \Phi_{k}(x, p, t)\right|^{2} .
$$


A particle is the approximation to a Dirac measure using some type of cutoff function [24]. The initial distribution

$$
\Phi_{0}(x, p)=\iint \Phi_{0}\left(x^{\prime}, p^{\prime}\right) \delta\left(x-x^{\prime}\right) \delta\left(p-p^{\prime}\right) d x^{\prime} d p^{\prime}
$$

is approximated by a linear combination of Dirac measures

$$
\Phi_{0}^{h}=\sum_{j=1}^{N} w_{j} \delta\left(x-x_{j}\right) \delta\left(p-p_{j}\right)
$$

for some set $\left\{x_{j}, p_{j}, w_{j}\right\}$ where $w_{j}>0$ are the weights and $N$ is the sample size. In a Monte Carlo approach, the position $\left(x_{j}, p_{j}\right)$ is randomly sampled from a distribution and sets $w_{j}=N^{-1} \int \Phi_{0}(x, p) d x d p$. In a deterministic approach, one assigns $\left(x_{j}, p_{j}\right)$ based on a uniform or nonuniform mesh and sets

$$
w_{j}=\int_{C_{j}} \Phi_{0}(x, p) d x d p
$$

for a cell $C_{j} \in \mathbb{R}^{d} \times \mathbb{R}^{d}$. To solve the Liouville equation, where $\delta\left(x(t)-x^{\prime}\right) \delta\left(p(t)-p^{\prime}\right)$ defines a single characteristic for the Hamiltonian $H(x, p)$, we solve the Hamiltonian system of equation (2.1) for each particle sampled from $f_{0}(x, p)$.

To determine $\Phi(x, p, t)$, solve $\dot{x}=p, \quad \dot{p}=-d V / d x$ with initial distribution $\Phi(x, p, 0)=\Phi_{0}(x, p)$ and interface conditions

$$
\hat{r} \Phi_{1, \text { out }}+\hat{t} \Phi_{2, \text { out }}=\Phi_{1, \text { in }} .
$$

The transmission and reflection at the interfaces can be handled either in a deterministic manner by tracking all paths or with a Monte Carlo routine by randomly choosing a path.

The deterministic method is the direct numerical adaption of the model (4.7) and every barrier interaction adds an additional path to follow. While single or double barrier problems can be efficiently implemented with a deterministic routine, tracking every path required for three or more barriers is impractical.

Rather than take every branch, the Monte Carlo approach instead takes one branch (either transmission or reflection) at each barrier intersection, by sampling from a uniform distribution $\xi \in[0,1]$. Take the transmission branch if

$$
\xi<\frac{|\hat{t}|}{|\hat{t}|+|\hat{r}|} .
$$

Otherwise, take the reflection branch. To incorporate the phase shift and conserve total mass, the weight after passing the barrier is set to

$$
w_{\text {out }}=(|\hat{t}|+|\hat{r}|) e^{i \theta} w_{\text {in }}
$$

where

$$
e^{i \theta}= \begin{cases}\hat{r} /|\hat{r}|, & \text { for reflection } \\ \hat{t} /|\hat{t}|, & \text { for transmission }\end{cases}
$$

The expected weight for a transmitted particle, determined from (4.11), (4.12) and (4.13) using the law of large numbers, is $\hat{t} w_{\text {in }}$. Similarly, the expected weight for reflected particle is $\hat{r} w_{\text {in }}$. This agrees with the interface condition (4.10). 
The solution $\Phi(x, p, t)=\iint_{-\infty}^{\infty} w \delta(x-\tilde{x}, p-\tilde{p}, t) d \tilde{x} d \tilde{p}$ is reconstructed by interpolating over a uniform mesh using a smoothing kernel such as a bicubic spline. Let $\Delta x$ denote the mesh spacing and let the nearest mesh point to $x$ be $x_{i}$ for some $i$. Let $r=\left(x-x_{i}\right) / \Delta x$ denote the offset from that mesh point. For $l \in\{-2, \ldots, 2\}$ define the mesh-constrained approximation to $\Phi(x, p, t)$ as

$$
\tilde{\Phi}_{i+1}(p, t)=w \sigma(r, l) / \Delta x
$$

with the cubic b-spline cut-off function [17]

$$
\sigma(r, l)= \begin{cases}\frac{1}{384}(2 r-1)^{4} & l=-2 \\ \frac{19}{96}-\frac{11}{24} r+\frac{1}{4} r^{2}+\frac{1}{6} r^{3}-\frac{1}{6} r^{4} & l=-1 \\ \frac{115}{192}-\frac{5}{8} r^{2}+\frac{1}{4} r^{4} & l=0 \\ \frac{19}{96}+\frac{11}{24} r+\frac{1}{4} r^{2}-\frac{1}{6} r^{3}-\frac{1}{6} r^{4} & l=1 \\ \frac{1}{384}(2 r+1)^{4} & l=2\end{cases}
$$

The semiclassical Monte Carlo algorithm can be summarized as follows:

0. Initialization. Compute the complex scattering coefficients associated with each momentum incident to each quantum barrier by using transfer matrix method $[1,13,6]$ or transmitting boundary method [15].

1. Choose an initial particle $x_{0}$ from the initial distribution using Monte Carlo sampling.

2. For each particle, while $t^{n}<t^{\max }$

(a) Compute the trajectory in the classical region either analytically or using a symplectic solver.

(b) At a barrier use a uniform random sampling to determine reflection or transmission. Use (4.11) to decide transmission or reflection and use (4.12) to incorporate phase shift and mass conservation.

(c) Take $p=-p$ for reflection and take $p=q=p \sqrt{1-2 \Delta V / p^{2}}$ for transmission.

3. Reconstruct the solution using (4.14). Go back to step 1.

\section{Examples}

In this section, we present four examples to validate the coherent semiclassical model. We consider a harmonic oscillator with a delta-function barrier, a resonant double-delta potential, a resonant multiple delta potential, and a rectangular barrier. Examples 1, 2 and 4 are solved analytically using a Lagrangian method in order to compare the convergence of the solutions to the Schrödinger equation to the the solution to the semiclassical model in the $\varepsilon \rightarrow 0$ limit. Example 2 is also solved numerically using the Lagrangian Monte Carlo scheme. Example 3 is solved numerically using the Eulerian scheme an analytical solution is infeasible. For all examples, we take the effective mass $m=1$.

5.1. Harmonic oscillator with delta-function barrier. Consider the harmonic oscillator with a delta-function barrier

$$
V(x)=\frac{1}{2} x^{2}+\varepsilon \alpha \delta(x) .
$$


and the initial conditions

$$
\begin{aligned}
\psi_{0}(x) & =\left(\pi \sigma^{2}\right)^{-1 / 4} \exp \left(-\frac{x-x_{0}}{2 \sigma^{2}}\right) \exp \left(i \varepsilon^{-1} p_{0} x\right), \\
f_{0}(x, p) & =(\pi \sigma)^{-1} \exp \left(-\frac{\left(x-x_{0}\right)^{2}}{\sigma^{2}}\right) \delta\left(p-p_{0}\right), \\
\Phi_{0}(x, p) & =(\pi \sigma)^{-1 / 2} \exp \left(-\frac{\left(x-x_{0}\right)^{2}}{2 \sigma^{2}}\right) \delta\left(p-p_{0},\right)
\end{aligned}
$$

for the Schrödinger equation, the decoherent semiclassical model, and the coherent semiclassical model, respectively. The initial density distribution

$$
\rho_{0}(x)=\left|\psi_{0}(x)\right|^{2}=\int_{-\infty}^{\infty} f_{0}(x, p) d p=\int_{-\infty}^{\infty}\left|\Phi_{0}(x, p)\right|^{2} d p
$$

is given by

$$
\rho_{0}(x)=(\pi \sigma)^{-1} \exp \left(-\frac{\left(x-x_{0}\right)^{2}}{\sigma^{2}}\right) .
$$

for each of the initial conditions. To simplify the solution, choose $x_{0}$ so that $\rho_{0}(x)$ is negligible for $x<0$.

The coherent and thin-barrier models are solved analytically using the Lagrangian formulation, tracking the trajectories backwards in time and branching characteristics at each barrier. For a simple harmonic oscillator $V(x)=\frac{1}{2} x^{2}$, the semiclassical solution is the rotation of the initial distribution about the origin in phase space by an angle $t-\tan ^{-1}(p / x)$. Because the trajectory is closed, we may disregard the phase changes from the harmonic oscillator and only track the phase changes from the delta potential barrier. The decoherent and coherent position densities are calculated from (4.8) and (4.9) by taking the projection of the characteristic onto the $x$-axis:

$$
\begin{aligned}
\text { decoherent: } & \rho(x, t)=\frac{\rho_{0}(|x \sec t|)}{|\sec t|} \sum\left|\left(\begin{array}{c}
m \\
n
\end{array}\right) \frac{(i \alpha x \sec t)^{n}}{\left(i \alpha x \sec t-\alpha^{2}\right)^{m}}\right|^{2} \\
\text { coherent: } & \rho(x, t)=\frac{\rho_{0}(|x \sec t|)}{|\sec t|}\left|\sum\left(\begin{array}{c}
m \\
n
\end{array}\right) \frac{(i \alpha x \sec t)^{n}}{\left(i \alpha x \sec t-\alpha^{2}\right)^{m}}\right|^{2}
\end{aligned}
$$

with the Hamiltonian $E=\frac{1}{2}\left(p^{2}+x^{2}\right)$. The sums are taken over even indices $n=$ $0,2, \ldots, m$ if $x>0$ and over odd indices $n=1,3, \ldots, m$ if $x<0$ where $m$ is the number of full half-periods of revolution (the integer part of $t / \pi+1 / 2$ ).

The scattering matrix for delta potential $V(x)=\varepsilon \alpha \delta(x)$ is [27]

$$
\mathrm{S}=\left(\begin{array}{ll}
\hat{r}_{1} & \hat{t}_{2} \\
\hat{t}_{1} & \hat{r}_{2}
\end{array}\right)=\left(\begin{array}{ll}
\frac{-i \alpha}{i \alpha+|p|} & \frac{|p|}{i \alpha+|p|} \\
\frac{p}{i \alpha+|p|} & \frac{-i \alpha}{i \alpha+|p|}
\end{array}\right)=\left(\begin{array}{cc}
\frac{-i \alpha}{i \alpha+\sqrt{2 E}} & \frac{p}{i \alpha+\sqrt{2 E}} \\
\frac{2 E}{i \alpha+\sqrt{2 E}} & \frac{-i \alpha}{i \alpha+\sqrt{2 E}}
\end{array}\right) .
$$

The quantum classical scattering coefficients are $R=|\hat{r}|^{2}=\alpha^{2} /\left(p^{2}+\alpha^{2}\right)$ and $T=|\hat{t}|^{2}=$ $p^{2} /\left(p^{2}+\alpha^{2}\right)$.

The Schrödinger equation is solved numerically using the Crank-Nicolson scheme

$$
\psi\left(x_{i}, t+\Delta t\right)=\left(I+\frac{1}{2} i \varepsilon^{-1} \Delta t \mathrm{H}\right)^{-1}\left(I-\frac{1}{2} i \varepsilon^{-1} \Delta t \mathrm{H}\right) \psi\left(x_{i}, t\right)
$$


where the discrete Hamiltonian operator

$$
\mathrm{H}_{i j}=\frac{-\varepsilon^{2}}{2} \frac{\delta_{i, j-1}-2 \delta_{i j}+\delta_{i, j+1}}{(\Delta x)^{2}}+V\left(x_{i}\right) \delta_{i j}
$$

with Kronecker delta $\delta_{i i}=1$ and $\delta_{i j}=0$ if $i \neq j$. The delta-function is approximated as $1 / \Delta x$ if $x_{i}=0$ and 0 otherwise, for a meshsize $\Delta x$. In order to guarantee correct approximation to physical observables for small $\varepsilon$ using the Crank-Nicolson scheme, one needs to take $\Delta x=o(\varepsilon)$ and $\Delta t=o(\varepsilon)[20]$.

The scattering matrix $\mathrm{S}$ given in (5.3) is a unitary matrix which rotates an input vector by $-i \tan ^{-1}(\alpha / 2 E)$. We computed the solutions a delta-potential of strength $\alpha=\sqrt{3}$, which permits $25 \%$ transmission. The Schrödinger equation is solved using grid spacing and time steps $\Delta x=\Delta t=5 \times 10^{-5}$. The $l^{1}$-errors for solutions at time $t=1.85$ are listed in Table 5.1. The convergence rate of the $l^{1}$-error is about 1.4 as $\varepsilon \rightarrow 0$.

The harmonic oscillator with delta-function barrier provides a good example of the entropy-preserving, time-reversible behavior of the coherent semiclassical model and the entropy increasing behavior of the decoherent thin-barrier model. See figure 5.1. Notice that over time the decoherent thin-barrier model approaches an equilibrium state with an equal distribution of the particle density to the right and left of the barrier. In contrast, the solutions to the coherent model and the Schrödinger equation do not approach an equilibrium state.

TABLE 5.1. Errors in solutions for different values of $\varepsilon$ for Example 5.1.

\begin{tabular}{lcccc}
\hline$\varepsilon$ & $50^{-1}$ & $100^{-1}$ & $200^{-1}$ & $400^{-1}$ \\
\hline$l^{1}$-error & 0.670 & 0.290 & 0.0969 & 0.0398 \\
\hline
\end{tabular}

5.2. Double delta potential. The resonant double-delta potential barrier

$$
V=\alpha \varepsilon[\delta(x-\ell / 2)+\delta(x+\ell / 2)]
$$

is an idealized double-barrier quantum well structure associated with resonant tunneling diodes (RTDs) [14, 21, 26]. An RTD consists of thin layers of different semiconductors which are sandwiched together. The length of the entire RTD structure is on the scale of a de Broglie wavelength. The region outside the barrier is doped to provide a sufficient number of free electrons. The transmission probability of an RTD is oscillatory and admits narrow peaks of total or almost total transmission well below the cutoff energy for classical transmission. By changing the strength $\alpha$ and separation $\varepsilon \ell$ of the barriers, the resonance may be tuned to admit electrons of varying energies. If the well is sufficiently wide, resonant particles are trapped in the well and slowly escape over time.

To compute the scattering coefficients for each barrier, we use the transfer matrices $\mathrm{M}=\mathrm{P}^{1 / 2} \mathrm{DP}^{1 / 2}$ where the transfer matrix for the delta potential $\mathrm{P}$ and the transfer matrix for the displacement $\mathrm{D}$ are given by

$$
\mathrm{P}=\left(\begin{array}{cc}
e^{i \ell p} & 0 \\
0 & e^{-i \ell p}
\end{array}\right) \quad \text { and } \quad \mathrm{D}=\left(\begin{array}{cc}
1+\alpha / i p & \alpha / i p \\
-\alpha / i p & 1-\alpha / i p
\end{array}\right)
$$

The scattering matrix is given by

$$
\mathrm{S}=\left(\begin{array}{cc}
r_{1} & t_{2} \\
t_{1} & r_{2}
\end{array}\right)=\left(\begin{array}{cc}
-m_{21} / m_{22} & 1 / m_{22} \\
\operatorname{det}(\mathrm{M}) / m_{22} & m_{12} / m_{22}
\end{array}\right),
$$


Decoherent Model
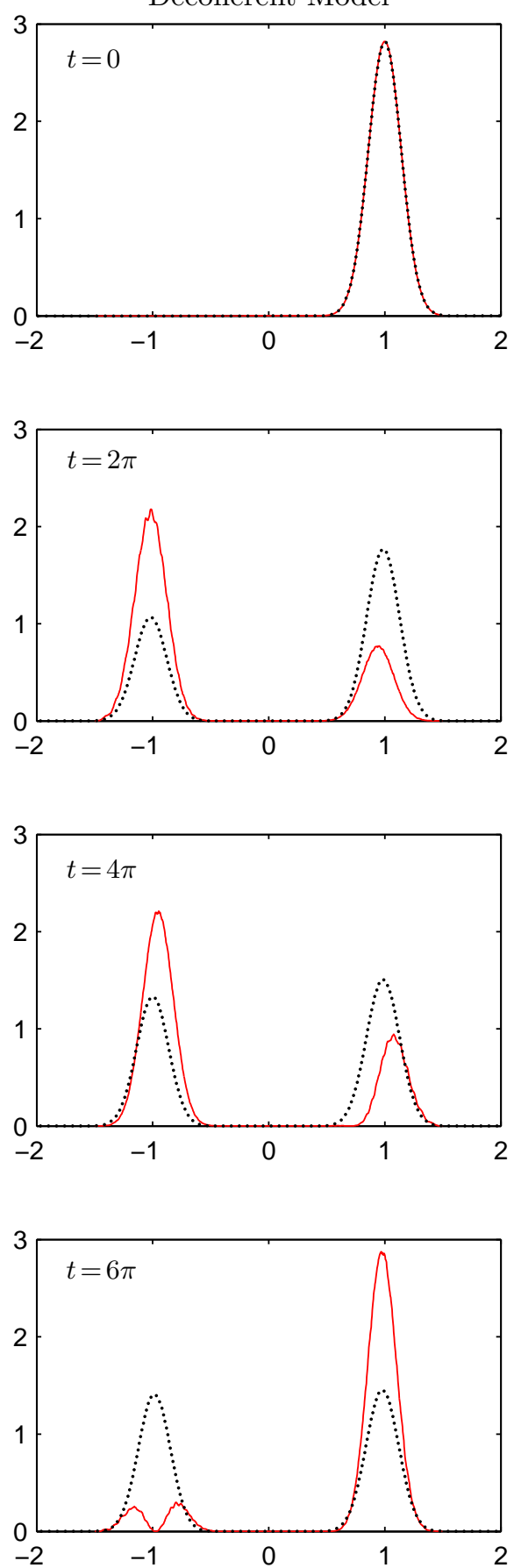

Coherent Model
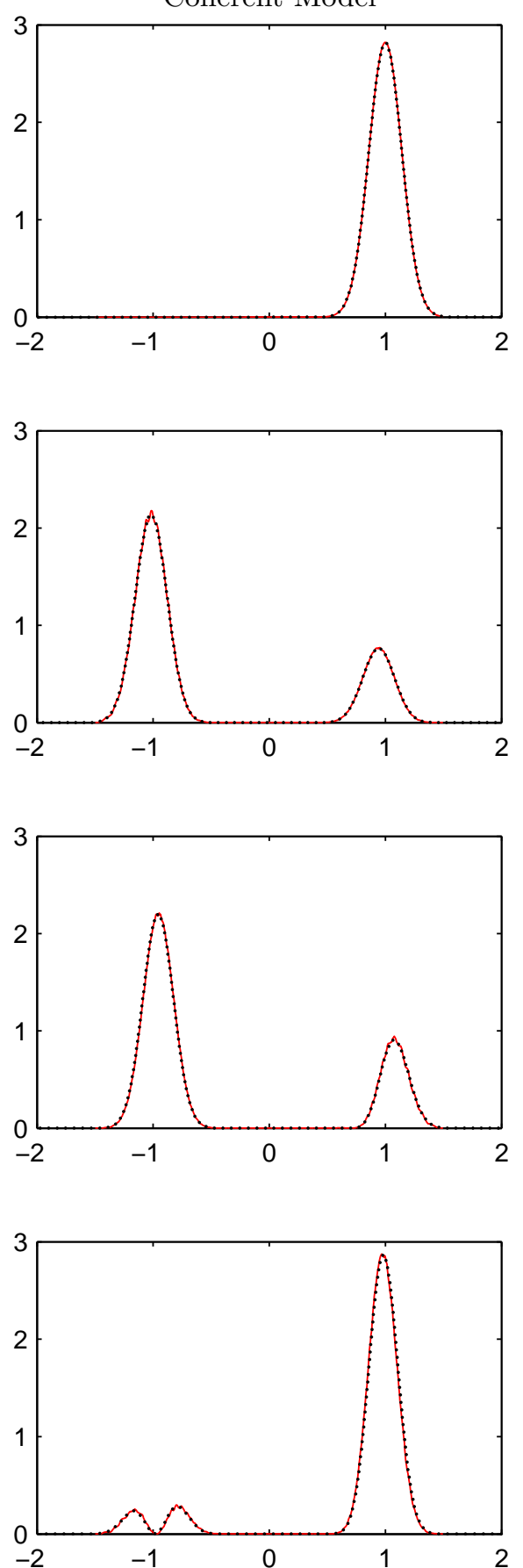

FIG. 5.1. Solution to Example 5.1 at $t=0,2 \pi, 4 \pi$ and $6 \pi$. The solid curve in each of the plots is the solution to the Schrödinger equation. The dotted curve is the solution to the semiclassical model. 
Thin-barrier Model

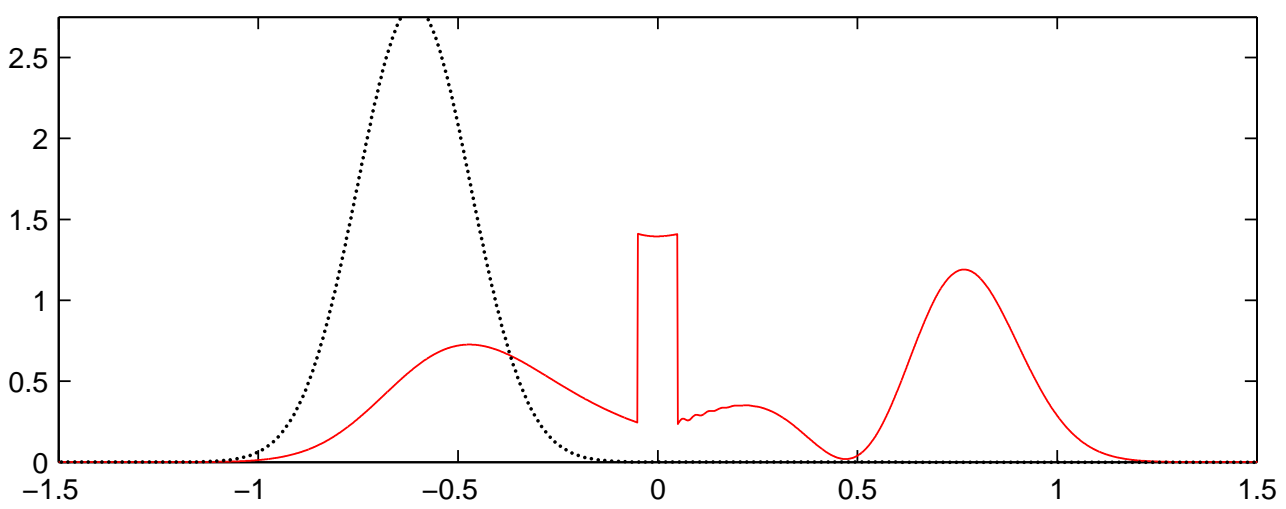

Decoherent Model

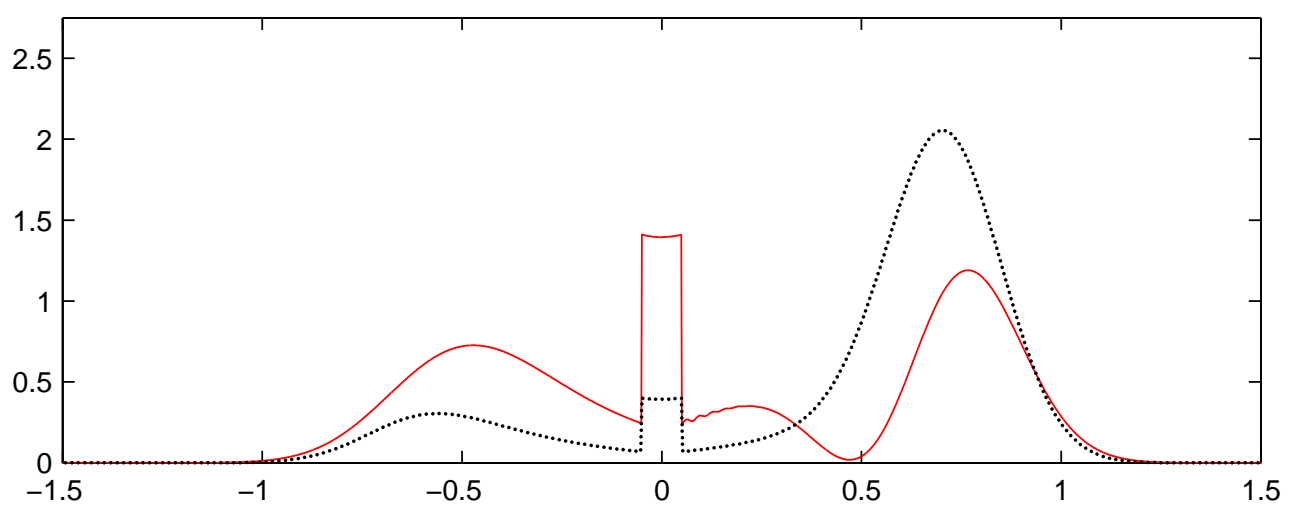

Coherent Model

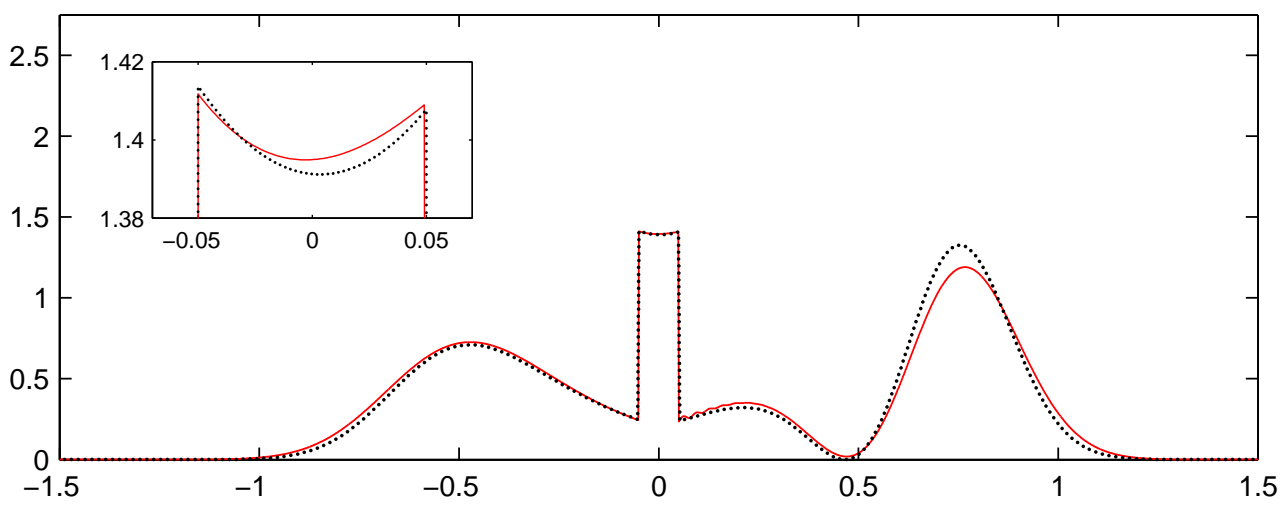

Fig. 5.2. Solution to Example 5.2 at $t=1.85$. The solid curve in each of the plots is the weak solution to the Schrödinger equation and the dotted curve is the solution to the semiclassical model. 


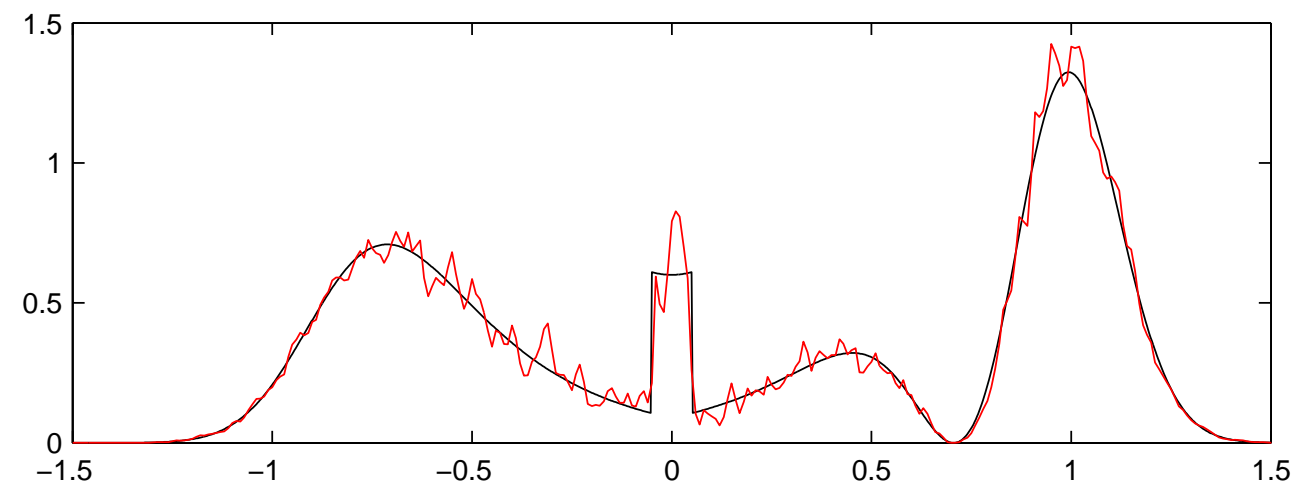

FIG. 5.3. Solution to the coherent semiclassical model of Example 5.2 at time $t=1.85$ using the Monte Carlo method using $10^{5}$ particles and the exact solution computed from the Schrödinger equation.

so the transmission and reflection coefficients are

$$
\begin{gathered}
\hat{t}=t_{1}=t_{2}=\frac{i|p|}{-\alpha+i|p|} e^{i|p| \ell} \\
\hat{r}=r_{1}=r_{2}=\frac{\alpha}{-\alpha+i|p|} e^{i|p| \ell} .
\end{gathered}
$$

The thin-barrier model combines the two delta potentials into a single barrier using the transfer matrix PDP, for which the transmission coefficient is

$$
T=\frac{p^{4}}{\left|\alpha^{2}\left(e^{2 i|p| \ell}-1\right)+2 i \alpha\right| p\left|+p^{2}\right|^{2}} .
$$

When $\alpha=-|p| \cot (\ell|p|)$, the barrier is resonant and admits total transmission $T=1$.

Consider the Gaussian initial conditions (5.1) with $p_{0}=-1, x_{0}=1$ and $\sigma=0.2$. Take $p_{0}=-1, \ell=10$, and $\alpha=-\cot (\ell)$. The Schrödinger equation was solved using the Crank-Nicholson scheme using grid spacing and time steps $\Delta x=\Delta t=5 \times 10^{-5}$. The semiclassical solution was computed analytically using the general solution presented in Example 5.4. The semiclassical solution was also computed numerically to verify the Monte Carlo Lagrangian algorithm.

The solutions are plotted in figure 5.2 at time $t=1.85$. The first plot compares the thin-barrier model using a single barrier with the solution of the Schrödinger equation. This approximation is good when the barrier separation is sufficiently small, and it clearly fails in this example. The second plot compares the solution of the decoherent model with the solution of the Schrödinger equation. The decoherent model treats each delta barrier as a separate thin barrier but does not include complex phase information. The third plot compares solution of the coherent model, which uses the Liouville equation between two delta-function potentials, with the solution of the Schrödinger equation The intrabarrier solution to the Schrödinger equation is highly oscillatory because of interference of reflected and transmitted waves. In each of the three plots, the "weak" (or averaged) solution to the Schrödinger equation is depicted. The "weak" solution is computed by averaging the upper and lower envelopes of the solutions to the Schrödinger equation. The upper and lower envelopes 
are approximated by cubic spline interpolation by using the relative extrema of the oscillations as knots for the spline.

The $l^{1}$-difference between the solutions to Schrödinger solution and the solution to the coherent semiclassical model at time $t=1.85$ are listed in Table 5.2. The convergence rate of the $l^{1}$-error is about 1.0 as $\varepsilon \rightarrow 0$.

TABlE 5.2. Errors in solutions for different values of $\varepsilon$ for Example 5.2.

\begin{tabular}{lcccc}
\hline$\varepsilon$ & $50^{-1}$ & $100^{-1}$ & $200^{-1}$ & $400^{-1}$ \\
\hline$l^{1}$-error & 0.306 & 0.166 & 0.080 & 0.0362 \\
\hline
\end{tabular}

5.3. Multiple delta potentials. A natural extension to the two barrier example is a multiple barrier example. Such a potential, similar to the Kronig-Penney model, approximates the Coulomb potentials of atoms in a crystalline lattice. In this case, we use eleven equally spaced delta function potentials,

$$
V(x)=\sum_{j=-5}^{5}-\varepsilon \delta(x-20 j \varepsilon),
$$

with $\varepsilon=200^{-1}$. The initial conditions are given by (5.1) with $x_{0}=1.25, \sigma=0.2$, and $p_{0}=-1$. The coherent semiclassical model is solved numerically using the finitevolume method detailed in section 4.1. See figure 5.4. The $l^{1}$-error in the numerical solution at $t=2.0$ is approximated by comparing the numerical solution with $N$ meshpoints in spaces and $N$ time steps with the numerical solution with 6400 points in space and steps in time. The error is given in Table 5.3. The convergence rate of the $l^{1}$-error is about 1.25 as $\Delta x, \Delta t \rightarrow 0$.

TABLE 5.3. Errors in solutions for different mesh sizes for Example 5.3.

\begin{tabular}{lcccc}
\hline$N$ & 100 & 200 & 400 & 800 \\
\hline$l^{1}$-error & 0.207 & 0.087 & 0.036 & 0.015 \\
\hline
\end{tabular}

5.4. Two step potentials. Finally, we consider the solution of the pure-state Schrödinger equation with rectangular barrier formed by two step potentials

$$
V(x)= \begin{cases}V_{1}, & x \in \mathcal{C}_{1}=(-\infty, 0) \\ V_{2}, & x \in \mathcal{C}_{2}=(0, L) \\ V_{3}, & x \in \mathcal{C}_{3}=(L, \infty)\end{cases}
$$

where $L=\frac{1}{10}, V_{1}=0, V_{2}=\frac{1}{2}$, and $V_{3}=-1$.

We compute the analytical solution to the semiclassical model by using the method of characteristics. We simplify the solution by taking the initial conditions 

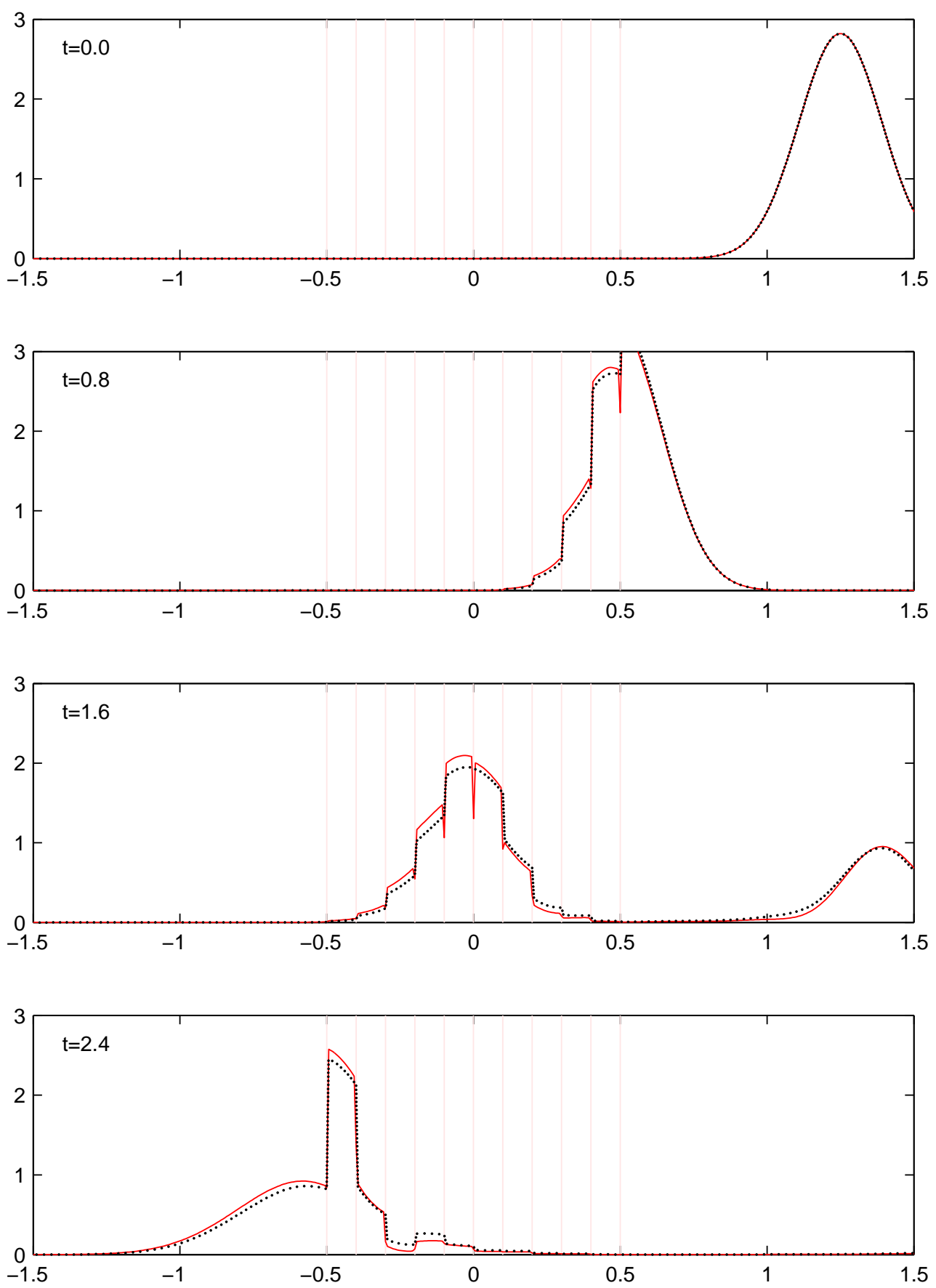

FIG. 5.4. Solution to the coherent semiclassical model of Example 5.2 at times $t=0,0.8,1.6$ and 2.4 using the finite volume approach (dotted curve) along with the averaged solution to Schrödinger equation (solid curve). 
$\Phi_{0}(x, p)=0$ for $x>0$. Let $L=\varepsilon \ell$. Then $\Phi(x, p, t)$ equals

$$
\begin{cases}\Phi_{0}\left(x-p_{1} t, p_{1}\right), & x \in \mathcal{C}_{1}, p>0, \\ \hat{r}_{12} \Phi_{0}\left(-x-p_{1} t,-p_{1}\right)+ & x \in \mathcal{C}_{1}, p<0, \\ \sum_{n \geq 0} \hat{t}_{12} \hat{t}_{21} \hat{r}_{23}\left(\hat{r}_{23} \hat{r}_{21}\right)^{n} \Phi_{0}\left(-x-p_{1} t+\frac{p_{1}}{p_{2}}(n+1) 2 L, p_{1}\right), & x \in \mathcal{C}_{2}, p>0, \\ \sum_{n \geq 0}^{n} \hat{t}_{12}\left(\hat{r}_{23} \hat{r}_{21}\right)^{n} \Phi_{0}\left(\frac{p_{1}}{p_{2}} x-p_{1} t+\frac{p_{1}}{p_{2}} n 2 L, p_{1}\right), & x \in \mathcal{C}_{2}, p<0, \\ \sum_{n \geq 0}^{n} \hat{r}_{23} \hat{t}_{12}\left(\hat{r}_{23} \hat{r}_{21}\right)^{n} \Phi_{0}\left(-\frac{p_{1}}{p_{2}}(L-x)+\frac{p_{1}}{p_{2}}(2 n+1) L-p_{1} t, p_{1}\right), & x \in \mathcal{C}_{3}, p>0, \\ \sum_{n \geq 0}^{\hat{t}_{12}} \hat{t}_{23}\left(\hat{r}_{23} \hat{r}_{21}\right)^{n} \Phi_{0}\left(\frac{p_{1}}{p 3}(x-L)+\frac{p_{1}}{p_{2}}(2 n+1) L-p_{1} t, p_{1}\right), & x \in \mathcal{C}_{3}, p<0, \\ 0, & \end{cases}
$$

where the $\hat{t}_{i j}$ denotes the transmission coefficient for a particle from region $\mathcal{C}_{i}$ to $\mathcal{C}_{j}$ with $\hat{r}_{i j}$ denotes the reflection coefficient for a particle in region $\mathcal{C}_{i}$ with a barrier separating regions $\mathcal{C}_{i}$ and $\mathcal{C}_{j}$. The momenta in the three regions $\mathcal{C}_{j}$ are given by $p_{j}=\sqrt{p^{2}-2\left(V_{j}-V_{1}\right)}$. The initial distribution is

$$
\Phi_{0}(x, p)=\frac{1}{\Delta x}\left(\frac{p}{p_{j}} \int_{x-\Delta x / 2}^{x+\Delta x / 2} f_{0}(x, p) d x\right)^{1 / 2} .
$$

To compute the scattering coefficients, we decompose the well into a barrier located at $x=0$ and a barrier located at $x=L$. The transfer matrices associated with a potential jump and the displacement $\varepsilon \ell / 2$ are

$$
\mathrm{P}_{i j}=\frac{1}{2}\left(\begin{array}{ll}
1+p_{i} / p_{j} & 1-p_{i} / p_{j} \\
1-p_{i} / p_{j} & 1+p_{i} / p_{j}
\end{array}\right) \quad \text { and } \quad \mathrm{D}_{i}=\left(\begin{array}{cc}
e^{i \ell p_{i} / 2} & 0 \\
0 & e^{-i \ell p_{i} / 2}
\end{array}\right) .
$$

The associated quantum scattering matrix for the transfer matrix $\mathrm{D}_{i} \mathrm{P}_{i j} \mathrm{D}_{j}$ is

$$
\mathrm{S}_{i j}=\frac{1}{p_{i}+p_{j}}\left(\begin{array}{cc}
\left(p_{i}-p_{j}\right) e^{i \ell p_{i}} & 2 p_{j} e^{i \ell\left(p_{i}+p_{j}\right) / 2} \\
2 p_{i} e^{i \ell\left(p_{i}+p_{j}\right) / 2} & \left(p_{j}-p_{i}\right) e^{i \ell p_{j}}
\end{array}\right) .
$$

From (3.10), the associated semiclassical scattering coefficients are

$$
\hat{r}_{i j}=\frac{p_{i}-p_{j}}{p_{i}+p_{j}} e^{i 2 \ell p_{i}} \quad \text { and } \quad \hat{t}_{i j}=\frac{2 \sqrt{p_{i} p_{j}}}{p_{i}+p_{j}} e^{i \ell\left(p_{i}+p_{j}\right)} .
$$

We computed the solutions to the Schrödinger equation and semiclassical model at time $t=1.5$ for a distribution centered at $\left(x_{0}, p_{0}\right)=(-1,1.08)$ with spread $\sigma=0.2$. The Schrödinger equation is solved using the Crank-Nicolson scheme with $\Delta x=\Delta t=$ $5 \times 10^{-5}$. The solutions are plotted in figure 5.5. The solution to the Schrödinger equation exhibits oscillations in region $\mathcal{C}_{2}$ due to interference from reflected and transmitted waves. The weak limit in region $\mathcal{C}_{2}$ is approximated by averaging the upper and lower envelopes of the solutions when $\varepsilon=400^{1}$ and $800^{-1}$. The $l^{1}$-difference in the solutions at time $t=1.5$ are listed in Table 5.1. The convergence rate of the $l^{1}$-error is about 1.5 as $\varepsilon \rightarrow 0$. 

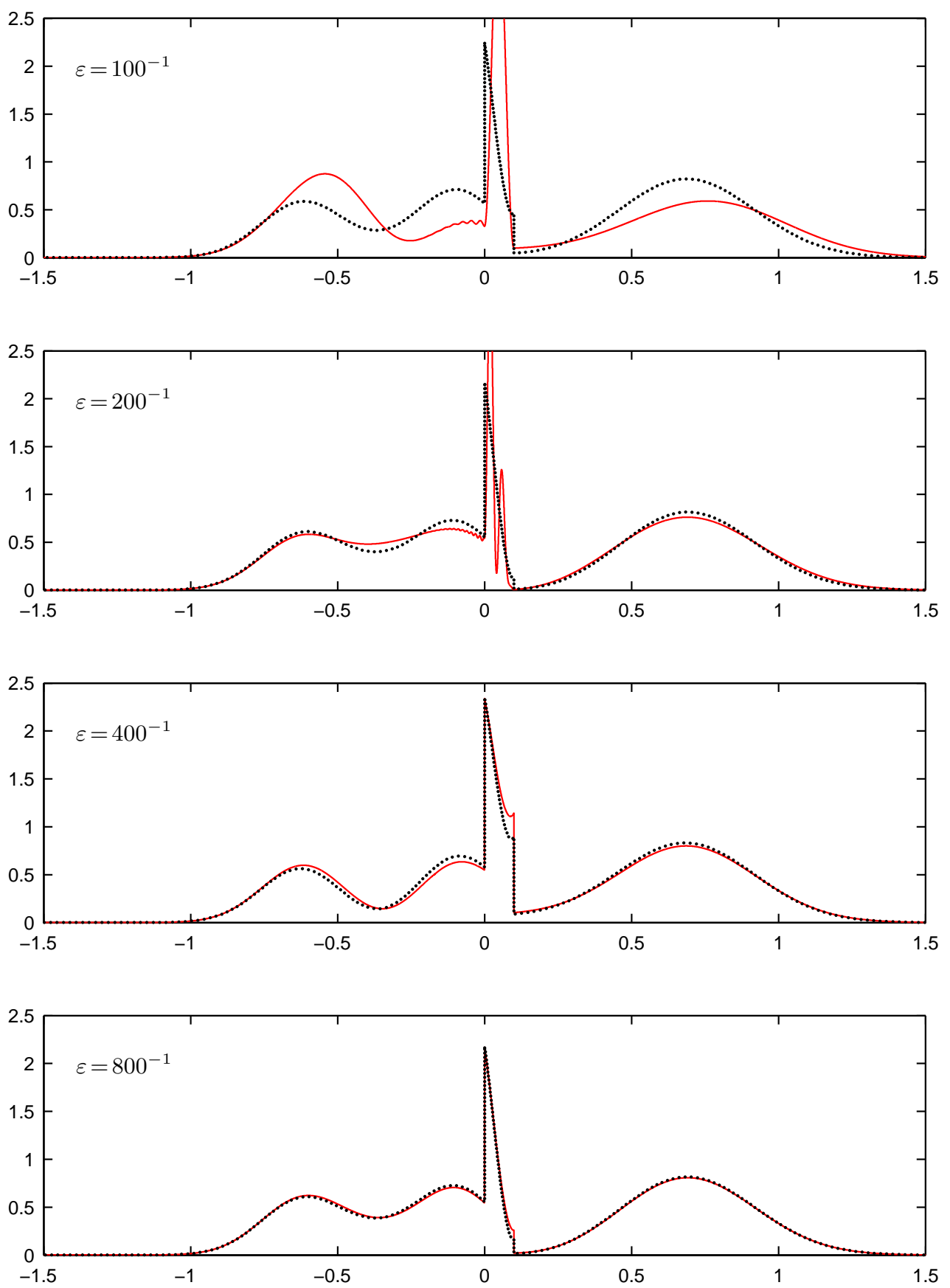

FiG. 5.5. Solution to Example 5.4 at time $t=1.5$. The solid curve is the solution to the Schrödinger equation. The dotted curve is the solution to the coherent semiclassical model. The solutions to the Schrödinger equation for $\varepsilon=200^{-1}$ and $400^{-1}$ are highly oscillatory in $[0,0.1]$ and the "weak" limits are computed by averaging the upper and lower solution envelopes. 
TABLE 5.4. Errors in solutions for different values of $\varepsilon$ for Example 5.2.

\begin{tabular}{lllll}
\hline$\varepsilon$ & $100^{-1}$ & $200^{-1}$ & $400^{-1}$ & $800^{-1}$ \\
\hline$l^{1}$-error & 0.4537 & 0.1192 & 0.0615 & 0.0218 \\
\hline
\end{tabular}

\section{Conclusion}

In this article, we proposed a one-dimensional time-dependent semiclassical transport model that accurately describes the weak limit of the pure-state Schrödinger equations. This model extends the thin-barrier models introduced in $[7,8]$ by including phase information so that the model can treat problems in which quantum coherence is critical. This model uses the complex-valued Liouville equation with an interface condition determined using complex-valued quantum scattering coefficients. Both Eulerian and Lagrangian numerical implementations were introduced and several numerical examples, including resonant multiple barriers, demonstrated the validity of the model in capturing the semiclassical limit across quantum barriers where interference can occur.

In the future we will investigate multi-dimensional numerical implementation of the model. It is also interesting to seek a mathematical justification of this model as the semiclassical limit of the Schrödinger equation using some sort of Wigner transformation.

We also point out that the complex-valued Liouville equation has also been used recently to construct Gaussian beam approximations to the Schrödinger equation [12]. The Gaussian beam method is another asymptotic method for high frequency waves that is able to capture the correct phase shift at caustics.

\section{REFERENCES}

[1] Y. Ando and T. Itoh, Calculation of transmission tunneling current across arbitrary potential barriers, J. Appl. Phys., 61(4), 1497-1502, 1987.

[2] A. Arnold, Mathematical concepts of open quantum boundary conditions, Transport Theor. Stat., 30(4-6), 561-584, 2001.

[3] G. Bal, J.B. Keller, G. Papanicolaou and L. Ryzhik, Transport theory for acoustic waves with reflection and transmission at interfaces, Wave Motion, 30(4), 303-327, 1999.

[4] N. Ben Abdallah, P. Degond and I.M. Gamba, Coupling one-dimensional time-dependent classical and quantum transport models, J. Math. Phys., 43(1), 1-24, 2002.

[5] P. Gérard, P.A. Markowich, N.J. Mauser and F. Poupaud, Homogenization limits and Wigner transforms, Commun. Pure Appl. Math., 50(4), 323-379, 1997.

[6] P. Grossel and J.M. Vigoureux, Nonlocal approach to scattering in a one-dimensional problem, Phys. Rev. A, 50(5), 3627-3637, 1994.

[7] S. Jin and K.A. Novak, A semiclassical transport model for thin quantum barriers, Multiscale Model. Simul., 5(4), 1063-1086, 2006.

[8] S. Jin and K.A. Novak, A semiclassical transport model for two-dimensional thin quantum barriers, J. Comp. Phys., 226, 1623-1644, 2007.

[9] S. Jin and X. Wen, Hamiltonian-preserving schemes for the Liouville equation with discontinuous potentials, Commun. Math. Sci., 3(3), 285-315, 2005.

[10] S. Jin and X. Wen, Hamiltonian-preserving schemes for the Liouville equation of geometrical optics with discontinuous local wave speeds, J. Comp. Phys., 214, 672-697, 2006.

[11] S. Jin and X. Wen, Hamiltonian-preserving schemes for the Liouville equation of geometrical optics with partial transmissions and reflections, SIAM J. Numer. Anal., 44, 1801-1828, 2006.

[12] S. Jin, H. Wu and X. Yang, Gaussian beam methods for the Schrödinger equation in the semi-classical regime: Lagrangian and Eulerian formulations, Commun. Math. Sci., 6, 995-1020, 2008 
[13] B. Jonsson and S.T. Eng, Solving the Schrödinger equation in arbitrary quantum-well potential profiles using the transfer-matrix method, IEEE J. Quantum Elect., 726(11), 2025-2035, 1990.

[14] N.C. Kluksdahl, A.M. Kriman, D.K. Ferry and C.A. Ringhofer, Self-consistent study of the resonant tunneling diode, Phys. Rev. B., 39(11), 7720-7735, 1989.

[15] C.S. Lent and D.J. Kirkner, The quantum transmitting boundary method, J. Appl. Phys., 67(10), 6353-6359, 1990.

[16] R.J. Leveque, Finite Volume Methods for Hyperbolic Problems, Cambridge Texts in Applied Mathematics. Cambridge University Press, Cambridge, 2002.

[17] Shaofan Li and Wing Kam Liu, Meshfree Particle Methods, Springer-Verlag, Berlin, 2004.

[18] P.L. Lions and T. Paul, Sur les mesures de Wigner, Rev. Mat. Iberoamericana, 9(3), 553-618, 1993.

[19] V.A. Mandelstahm and H.S. Taylor, Spectral projection approach to the quantum scattering calculations, J. Chem. Phys., 102(19), 7390-7398, 1995.

[20] P.A. Markowich, P. Pietra and C. Pohl, Numerical approximation of quadratic observables of Schrödinger-type equations in the semi-classical limit, Numer. Math., 81(4), 595-630, 1999.

[21] P.A. Markowich, C.A. Ringhofer and C. Schmeiser, Semiconductor Equations, Springer-Verlag, Vienna, 1990

[22] A. Messiah, Quantum Mechanics. Vol. I, Translated from the French by G.M. Temmer. NorthHolland Publishing Co., Amsterdam, 1961.

[23] L. Miller, Refraction of high-frequency waves density by sharp interfaces and semiclassical measures at the boundary, J. Math. Pures Appl. (9), 79(3), 227-269, 2000.

[24] P.A. Raviart, An analysis of particle methods, Numerical Methods in Fluid Dynamics (Como, 1983), Lecture Notes in Math., Springer, Berlin, 1127, 243-324, 1985.

[25] L. Ryzhik, G.C. Papanicolaou and J.B. Keller, Transport equations for waves in a half space, Commun. Partial Differential Equations, 22(11-12), 1869-1910, 1997.

[26] J.P. Sun, G.I. Haddad, P. Mazumder and J.N. Schulman, Resonant tunneling diodes: Modes and properties, P. IEEE, 86(4), 641-661, 1998.

[27] J.S. Walker and J. Gathright, Exploring one-dimensional quantum-mechanics with transfermatrices, American J. Physics, 62(5), 408-422, 1994.

[28] E. Wigner, On the quantum correction for thermodynamic equilibrium, Phys. Rev., 40(5), 749-759, 1932. 